\title{
The Status of GEO600
}

\author{
Harald Lück ${ }^{1,2}$, P. Aufmuth ${ }^{1}$, O.S. Brozek ${ }^{2}$, K. Danzmann ${ }^{1,2}$,
} A. Freise ${ }^{1}$, S. Goßler ${ }^{1}$, A. Grado ${ }^{1}$, H. Grote ${ }^{1}$, K. Mossavi ${ }^{2}$, V. Quetschke ${ }^{1}$, B. Willke ${ }^{1,2}$, K. Kawabe ${ }^{3}$, A. Rüdiger $^{3}$, R. Schilling ${ }^{3}$, W. Winkler ${ }^{3}$, Ch. Zhao ${ }^{3}$, K.A. Strain ${ }^{4}$, G. Cagnoli ${ }^{4}$, M. Casey ${ }^{4}$, J.Hough ${ }^{4}$, M.Husman ${ }^{4}$, P.McNamara ${ }^{4}$, G.P.Newton ${ }^{4}$, M.V.Plissi ${ }^{4}$, N.A. Robertson ${ }^{4}$, S. Rowan ${ }^{4}$, D.I. Robertson ${ }^{4}$, K.D. Skeldon ${ }^{4}$, C.I. Torrie ${ }^{4}$, H. Ward ${ }^{4}$, B.F.Schutz ${ }^{5}$, I. Taylor ${ }^{5}$, B.S. Sathyaprakash ${ }^{6}$

\begin{abstract}
GEO600, the German/British gravitational wave detector currently being built in northern Germany, used advanced optical technologies to obtain a sensitivity comparable with the other, bigger detectors currently being built $[1,2]$. The installation of the ultra-high-vacuum system has almost been completed and the Mode Cleaners are operational.
\end{abstract}

\section{INTRODUCTION}

Five large scale gravitational-wave detectors are currently being built worldwide [1-3]. Despite the small size of GEO600 $(600 \mathrm{~m})$ in comparison to LIGO $(4 \mathrm{~km})$ and VIRGO $(3 \mathrm{~km})$, GEO600 will achieve a comparable peak sensitivity. This is possible by using advanced optical technologies such as dual-recycling $[4,5]$. This paper describes the current status of the GEO600 project and gives an introduction into the technologies used to fight the important noise sources.

1) Institut für Atom- und Molekülphysik, Universität Hannover, Callinstrasse 38, 30167 Hannover

2) Max-Planck-Institut für Quantenoptik, Außenstelle Hannover, Callinstrasse 38, 30167 Hannover

3) Max-Planck-Institut für Quantenoptik, Hans-Kopfermann-Strae 1, D-85748 Garching, Germany

4) Physics 8 Astronomy, University of Glasgow, Glasgow G12 8QQ

5) Max-Planck-Institut für Gravitationsphysik, Albert-Einstein-Institut, Haus 5, Am Mühlenberg, 14476 Golm, Germany

6) Department of Physics and Astronomy, University of Wales Cardiff, P.O. Box 913, Cardiff, Wales, CF2 $3 Y B$.

CP523, Gravitational Waves: Third Edoardo Amaldi Conference, edited by S. Meshkov (1) 2000 American Institute of Physics 1-56396-944-0/00/\$17.00 


\section{THE BASICS OF GEO600}

Before the laser light enters the main interferometer it is sent through two successive mode cleaners to attenuate fluctuations of the laser beam.

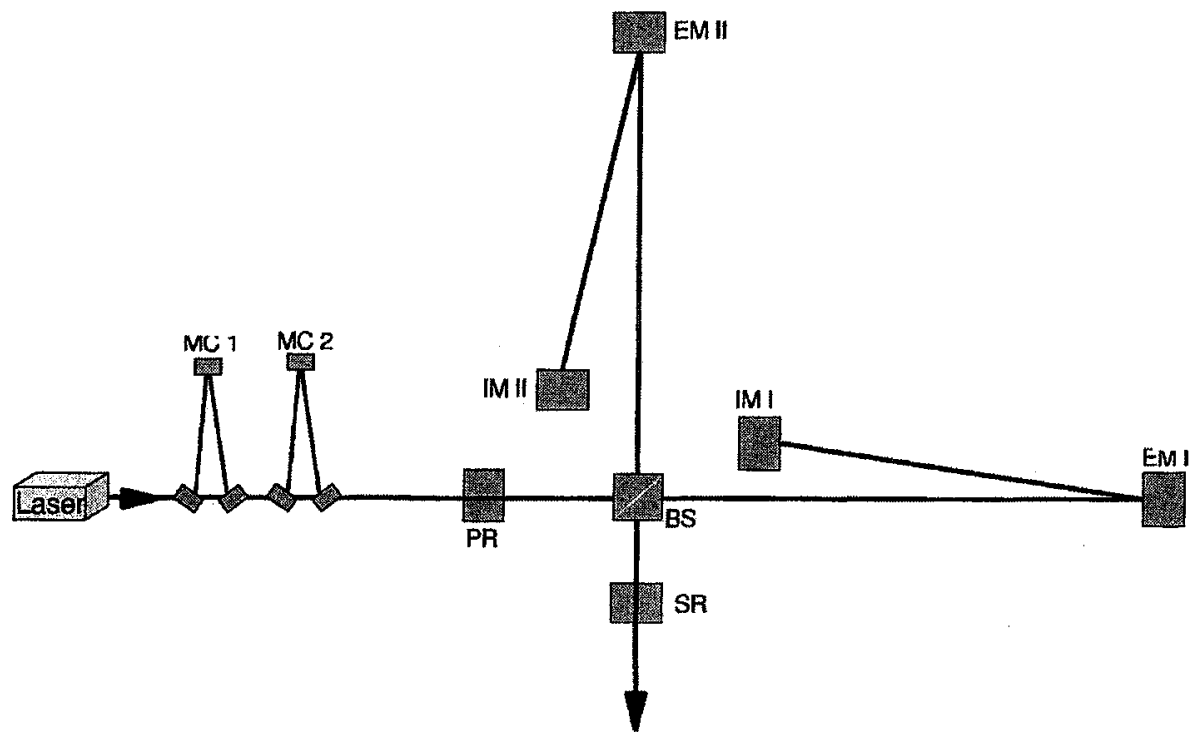

FIGURE 1. Optical layout of GEO600

The short arm length of GEO600 has to be compensated for by choosing a powerful optical layout. GEO600 uses a simple optical delay line which sends the light through the interferometer arms four times(DL4) to increase the optical round trip length to $2400 \mathrm{~m}$ (See Figure 1). The light is sent through the beam splitter (BS) to the end mirror (EM), is sent back to the inboard mirror (IM) and retraces it's incoming path back to the beam splitter. Figure 1 shows Power Recycling (PR) is employed to enhance the circulating light power by a factor of 2000 . Hence with a laser power of $10 \mathrm{~W}$ and an assumed loss of $50 \%$ through the mode cleaner the laser power available inside the interferometer at the beam-splitter will be $10 \mathrm{~kW}$. To further boost up the sensitivity Signal Recycling (SR) will be used. Signal Recycling reduces the shot noise in the vicinity of a tunable frequency and sacrifices sensitivity elsewhere. The spectral width of the sensitivity enhancement can be chosen by altering the reflectivity of the Signal Recycling mirror. Doing this online by using a thermally tunable etalon as a Signal Recycling mirror is an option currently being investigated. The sensitivity aimed 
at is shown in Figure 2. In addition to enhanced sensitivity SR also reduces the amount of non-TEM00 modes exiting through the output port due to imperfect mirror surfaces or imperfect alignment (Mode Healing) [6] and thus improves the signal to noise ratio.
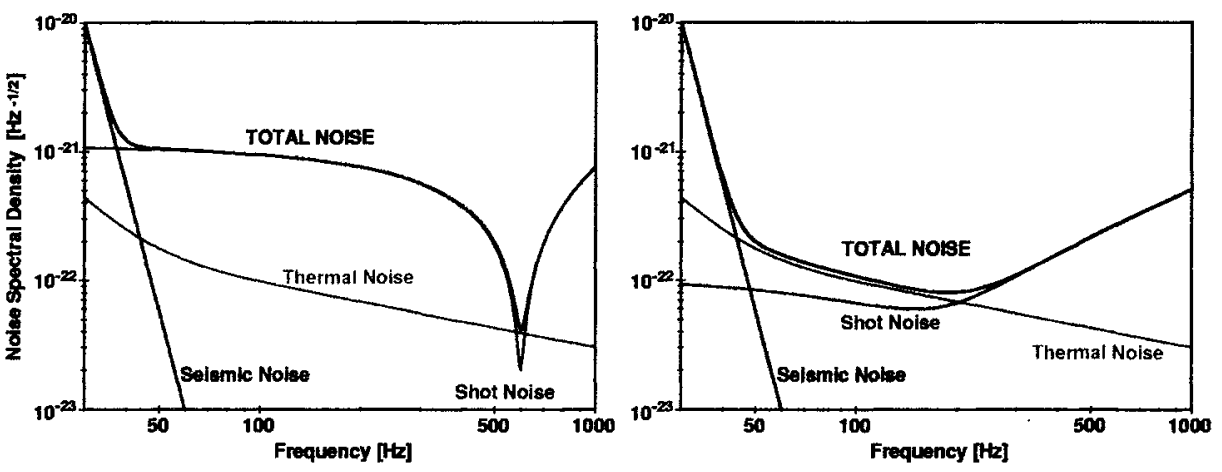

FIGURE 2. Sensitivity of GEO600 for broad- and narrow-band operation

The control signals for the differential Michelson length control and the Signal Recycling mirror will be obtained with Schnupp modulation from the output light and the light in one of the arms, respectively. This requires an arm length difference of about $10 \mathrm{~cm}$. The position of the Power Recycling mirror will be controlled with the Pound-Drever-Hall (rf-reflection locking) technique.

\section{STATUS OF THE INSTALLATION}

\section{Vacuum System}

To reach the desired sensitivity in GEO600 the residual gas pressure has to be kept below $10^{-8}$ mbar. Hydrocarbons can degrade the performance of the highly reflective mirrors at even lower pressures. Hence GEO600 uses an all-metal vacuum system in an attempt to reduce the hydrocarbon content below the sensitivity limit of the mass spectrometers, i.e. $10^{-14}$ mbar. With a volume of about $400 \mathrm{~m}^{3}$ the GEO600 vacuum system is currently the biggest UHV system in Europe.

The vacuum system of GEO600 is divided into 7 subsections:

- three tanks contain the two mode cleaner and injection optics. The mode cleaner tanks are separated from the central cluster by a $200 \mathrm{~mm}$ all metal gate valve.

- the central cluster of 4 tanks containing the Power Recycling mirror, beam splitter, compensation plate, and the inboard mirrors. The central cluster is set up, connected and pumped down (not yet baked) to a pressure in the upper $10^{-9} \mathrm{mbar}$ region with a small air leak left to be tracked down. 
- two beam tubes of $600 \mathrm{~m}$ length each, $60 \mathrm{~cm}$ diameter and a wall thickness of $0.8 \mathrm{~mm}$. Corrugated walls give the stability against the air pressure. The tubes are separated from the other sections by $600 \mathrm{~mm}$ all metal gate valves. The pressure in the beam tubes is in the upper $10^{-9} \mathrm{mbar}$ region. The tubes have received a bake at $200^{\circ} \mathrm{C}$ in air for two days and $250^{\circ} \mathrm{C}$ under vacuum for 5 days.

- two sections containing one end-tank each.

- two tanks containing the Signal Recycling mirror and output optics, separated from the central cluster by a $200 \mathrm{~mm}$ all metal gate valve to allow for a change of the Signal Recycling mirror without long down-times. These two tanks will be connected to the central cluster later on.

The whole vacuum system, except for the mode cleaner, is pumped by four magnetically levitated Turbo pumps with a pumping power of 10001/s (nitrogen) each backed by Scroll pumps $\left(25 \mathrm{~m}^{3} / \mathrm{h}\right)$.

\section{The Laser}

The laser for GEO600 is being developed in the Laser Zentrum Hannover. GEO600 uses an injection locked Nd-YAG master/slave system with the master being a stabilized diodepumped $1 \mathrm{~W}$ NPRO (Non-Planar-Ring-Oscillator) and the slave a diode end-pumped bow-tie ring resonator giving an output power of $13 \mathrm{~W}$. Currently the master laser is used on site for testing of the mode cleaner, whereas the master/slave system is still being optimized in the Hannover labs. At low frequencies the master laser is stabilized to a suspended rigid reference cavity made of ULE and it will be stabilized to the Power Recycling cavity in the measurement frequency band.

\section{Mode Cleaner}

GEO600 uses two mode cleaner in series to reduce fluctuations of the laser beam in shape, position and pointing. Each of the triangularly shaped mode cleaners has a round trip length of about $8 \mathrm{~m}$ and a Finesse of 1900 . Two successive mode cleaner have been implemented to obtain high attenuation values in short cavities with moderate Finesse.

Vacuum The mode cleaner section is pumped by a magnetically levitated Turbo pump (170l/s) backed by a scroll pump. Before the final bake the hydrocarbon content in the mode cleaner section was higher than expected (in the $10^{-11} \mathrm{mbar}$ region). In order not to contaminate the other vacuum sections which contain the main optics a $1 \mathrm{~cm}$ aperture will be inserted which limits the conductance to about 10l/s. Hence with a pumping power of $3000 \mathrm{l} / \mathrm{s}$ we can keep the hydrocarbon pressure in the central cluster in the $10^{-14}$ mbar region.

Optics The mirrors for the mode cleaners with a diameter of $100 \mathrm{~mm}$ and a thickness of $50 \mathrm{~mm}$ are made of fused silica (Suprasil1), polished by Rollei and ion-beam coated by the VIRGO group. 
Auto-alignment Both mode cleaner are equipped with an auto-alignment system which adjusts the mirrors with respect to the incoming beam. A similar auto-alignment system is described in [8]. The bandwidth is currently restricted to $0.3 \mathrm{~Hz}$, but will be increased to a few $\mathrm{Hz}$.

Suspension All the optics for both mode cleaner are suspended as double pendulums in two vacuum tanks $1 \mathrm{~m}$ in diameter each. One mirror in each mode cleaner is hung as a double pendulum with a reaction mass which itself also is suspended as a double pendulum.

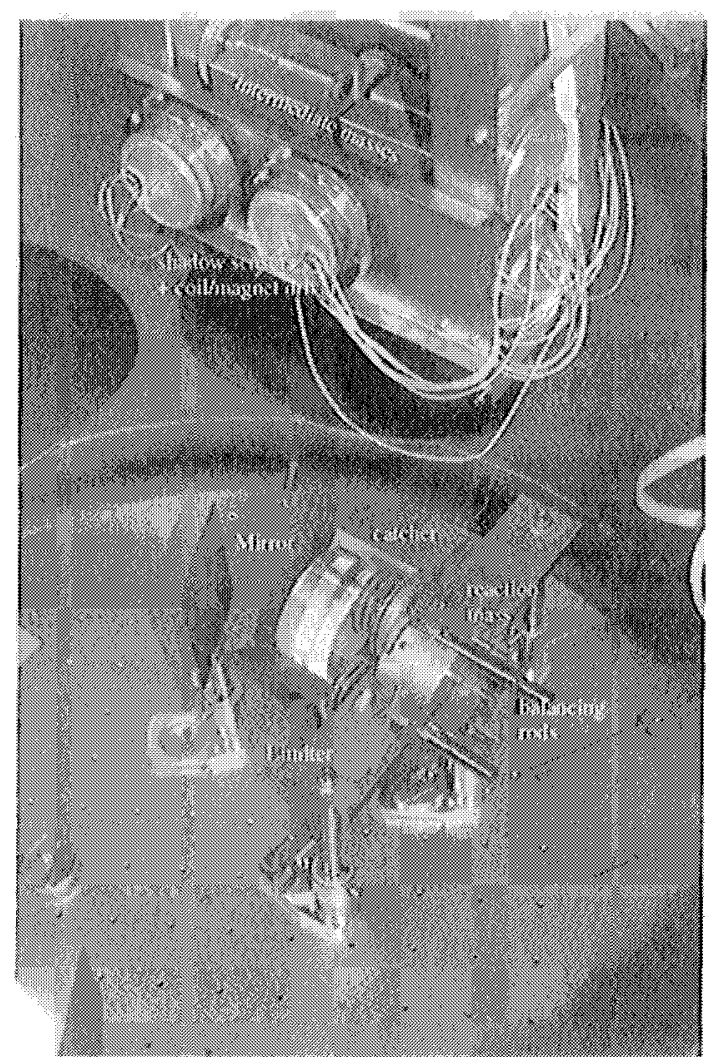

Fig. 3: Mode cleaner mirror suspended as a double-double pendulum (see Text)
Such a double-double pendulum can be seen in Figure 3 . The pendulum resonances are electronically damped at the intermediate masses using coil/magnet actuators. The motion with respect to a rigid frame is sensed with shadow sensors. Control forces for fast movements are applied between the mirror itself and the reaction mass. The magnets used here are directly glued onto the mirror surface using an inorganic glue. The current is supplied through bare $150 \mu \mathrm{m}$ copper wires. A $4 \mathrm{~cm}$ hole in the reaction mass allows to pass the laser beam pass through. The rods seen on the right hand side serve for balancing the reaction mass. Apropriately shaped stainless steel sheets limit the motion of the reaction mass such that is does not hit the mirror. Both the mirror and the reaction mass are suspended from stainless steel wires with a diameter of $60 \mu \mathrm{m}$ (cannot be seen in this picture.)

Status Both mode cleaner are installed and have been locked in air to the laser wavelength. The locking was robust and could be maintained for a few hours. The contrast at the input of the first and second mode cleaner was about $85 \%$ and $95 \%$, respectively. The mode matching into the first mode cleaner is not yet optimised. The throughput (about $60 \%$ ) of the 'mounting unit' between the two mode cleaner carrying two Farady isolators and an elctro optical modulator (24 surfaces) is not yet satisfactory and remains to be improved. 


\section{Main Interferometer}

\section{Vacuum}

The vacuum system, except for the end tanks, is finished. Small air leaks (internal or external) remain to be found but will not compromise the overall performance of GEO600.

\section{Suspension}

The suspension (see Figure 4) [9] of the mirrors has to ensure isolation of the mirrors against seismic disturbances and simultaneously allow microscopical movement and orientation. The first isolation stage in GEO600 is an active feedback stage consisting of a 3-dimensional piezoelectric actuator collocated with three geophones with a resonance frequency of $2 \mathrm{~Hz}$. A feedforward loop using an external $1 \mathrm{~Hz}$ geophone will improve the isolation to about $35 \mathrm{~dB}$. Atop of this active stage a passive rubber/stainless steel sandwich will give additional isolation above the resonance frequency of about $30 \mathrm{~Hz}$. A hexagonal frame structure (stack stabilizer) is supported by three of these stacks and connected to them via flex pivots for rotational freedom. Another hexagonal frame (rotational stage) rests on the stack stabilizer and can be rotated against it to prealign the mirror. Polished Alumina/Sapphire disks serve as a bearing. From the rotational stage the mirror is suspended as a triple pendulum with two additional cantilever spring stages for vertical isolation (see Figure 4). Careful design of the wire lengths and inclination and all moments of inertia ensure optimum coupling of the motion of all pendulum stages to the upper mass where all six degrees of freedom are damped by collocated sensor/actuator pairs. The lowest stage of the mirror suspension, will be a monolithic suspension entirely made of fused silica. 'Hydroxide-catalysis bonding' [10] attaches the mirrors to fibres which in turn are bonded to the stage above. The fast feedback to the end-mirrors for longitudinal locking uses electro-static actuators positioned on reaction masses with an identical suspension. After a successful design and prototyping phase the first main suspension is being installed on site.

\section{Optics}

- The main mirrors of the interfeometer (i.e. the end- and inboard mirrors) are made of fused silica (Suprasil 1) with a diameter (D) of $18 \mathrm{~cm}$ and a thickness (d) of $10 \mathrm{~cm}$. They are polished by General Optics and will be coated by the VIRGO group.

- The recycling mirrors are made of Suprasil 2 (a lesser grade for cost reasons) with $\mathrm{D}=15 \mathrm{~cm}$ and $\mathrm{d}=7.5 \mathrm{~cm}$.

- The beamsplitter requires special attention because it is the only optical element which is transmitted by the $10 \mathrm{~kW}$ light inside the cavity. Suprasil $311 \mathrm{SV}$ is a fused silica with especially low absorbtion (in this case the absorbtion was unmeasurable, i.e. below $0.5 \mathrm{ppm} / \mathrm{cm}$ ). 

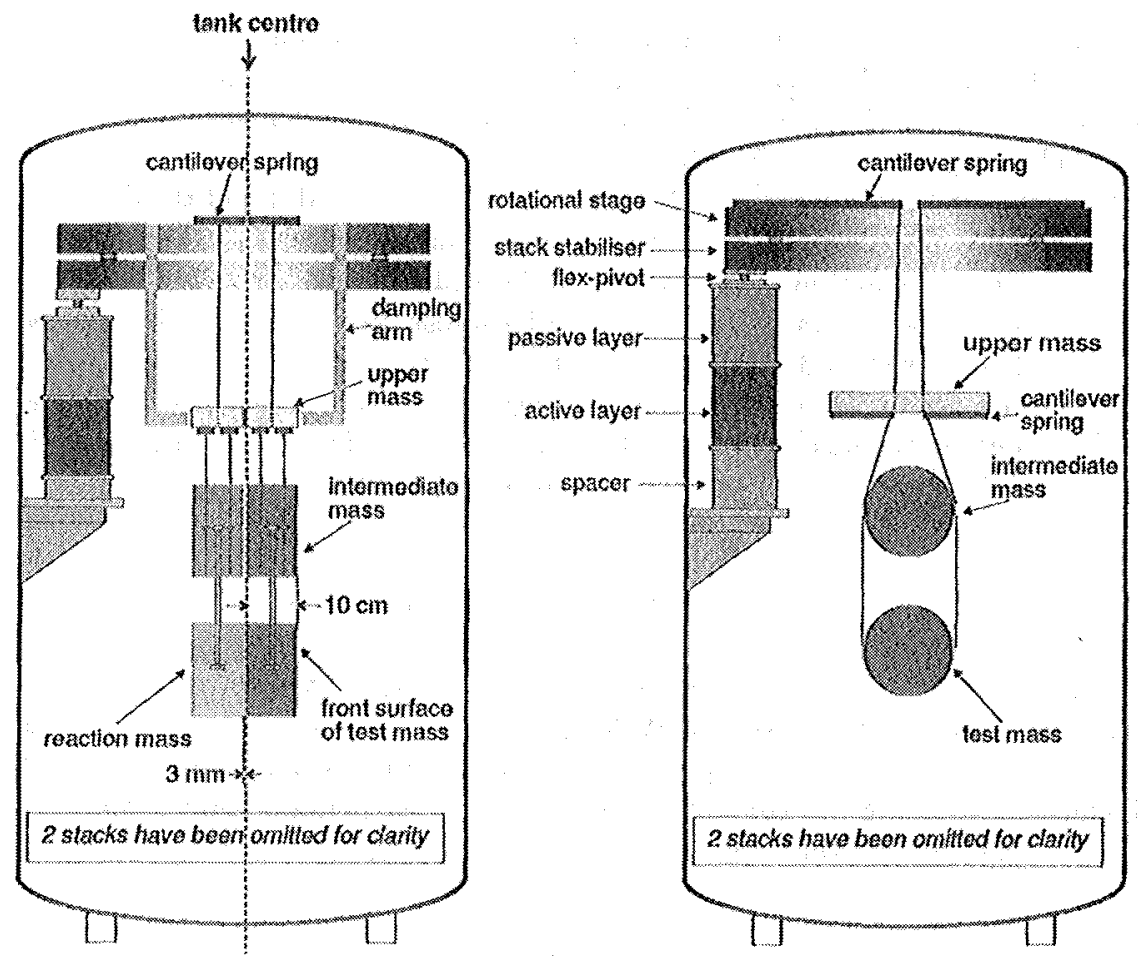

FIGURE 4. Side- and axial-view of the suspension for the main mirrors

- In the installation and debugging phase GEO600 will use test mirrors made from natural quarz hung in steel wire slings.

- The initial beam splitter will be made of Suprasil 312.

\section{Detector control}

Except for the active seismic isolation GEO600 uses analog feddback loops. Many of the loop parameters, e.g. gain or offset, are supervised by Labview software run on PCs. This allows remote control of mirror positions for prealignment purposes and gives short lock-aquisition times due to automatically setting optimum gains for each step.

\section{Data Aquisition}

The data aquisition system (DAQS) for GEO600 is based on the DAQS developed for LIGO but modified to the less demanding data rates of $0.5 \mathrm{MB} / \mathrm{s}$ in GEO600. 
Each of the three buildings will contain one data collection unit (DCU). The DCU is a VME crate running VxWorks-RTOS and Tornado Software and contains an 24-bit ADC interface capable of digitising 32 (16 for the end buildings) channels at a rate of $8 \mathrm{kHz}$ and 64 channels at a rate of $512 \mathrm{~Hz}$.

Debugging and optimisation of GEO600 will make extended use of the data aquisition system and use TRIANA [11] as a software tool. First look analysis of the data that does not require high computing power will be performed on site.

The data of three days will be buffered on hard disk on site and simultaneously transferred to Hannover via a $34 \mathrm{Mbit} / \mathrm{s}$ radiolink, where they will be stored by a tape robot. The whole data-set will by transferred over night via Internet to Potsdam where it will also be stored on tape. The tapes written in Hannover will be sent to Cardiff via mail. Hannover will create a subset of data to be distributed to other groups.

\section{Data Analysis}

Data analysis will be distributed between the Albert-Einstein-Institut in Golm/Potsdam, the University of Wales in Cardiff and Hannover. The software will run on Beowulf clusters with a capacity of 12 GFlops (Potsdam/Cardiff) and 6 GFlops (Hannover). Potsdam will concentrate on the search for periodic signals, Cardiff will do burst searches and the time critical analysis will be done in Hannover.

\section{PLANS}

Within the next few months the optics in one interferometer arm will be installed and operated as a $1200, \mathrm{~m}$ Fabry-Perot cavity to gain experience with the long interferometer arms. The second interferometer arm, power- and signal recycling will be installed subsequently. During these phases GEO600 will be operated using test optics hung in wire slings to avoid the risk of damaging the high quality optics. Data taking is expected to commence towards the end of 2001 .

\section{REFERENCES}

1. M. Coles, LIGO, this conference.

2. F. Marion, VIRGO, this conference.

3. K. Tsubono, M. Ando, TAMA, this conference.

4. B.J. Meers, Phys. Rev. D 38, pp. 2317-2326, (1988)

5. K.A. Strain, B.J. Meers, Phys. Rev. Lett 66, pp. 1391-1394, (1991)

6. G. Heinzel et al., Experimental demonstration of a Suspended Dual Recycling Interferometer for Gravitational Wave Detetction Phys. Rev. Lett. 81 pp. 5493-5496 (1998)

7. O.S Brozek et al., Proc. 18th Moriond Workshop, in press

8. G. Heinzel et al., Opt. Comm. 160, pp. 321-334, (1999) 
9. Design of Suspension System for GEO600, M.V. Plissi et al., Proceedings of 8th Marcel Grossman Meeting on General Relativity and Gravitation, Israel, ed. T Piran, World Scientific, pp. 1063-1065, (1999)

10. S. Rowan et al.

11. I. Taylor,

Documentation

for

Triana

OCL, http://www.astro.cf.ac.uk/pub/Ian.Taylor/Triana/index.html 